\title{
Estudo da viscosidade absoluta de soluções aquosas de F127 e sua influência no diâmetro hidrodinâmico de nanocompósitos AuNP-F127
}

\author{
Aline B. S. Santos (IC) ${ }^{1^{*}}$, Deizilene S. B. Gomes (PG) $)^{1,2}$, Maria A. G. Soler (PQ) ${ }^{1}$ \\ santos.alinebeatriz@gmail.com \\ ${ }^{1}$ Universidade de Brasília, Laboratório de Nanofilmes e Nano Dispositivos, Instituto de Física, Brasília- \\ DF, Brasil. \\ ${ }^{2}$ Instituto Federal de Educação, Ciência e Tecnologia de Rondônia, Ji-Paraná-RO, Brasil. \\ Palavras-chave: nanopartículas de ouro, Pluronic F127, diâmetro hidrodinâmico, viscosidade, \\ nanocompósito.
}

\section{Introdução}

O uso de nanomateriais se mostra cada vez mais importante, por causa de suas diversas aplicações no campo medicinal, na agricultura, meio ambiente, e na eletrônica. Tudo isso é possível devido às suas propriedades diferenciadas, tais como térmicas, eletrônicas, ópticas, magnéticas dentre outras. As nanopartículas de ouro estudadas, apresentam um baixo custo na sua produção, podem ser amplamente empregadas no campo medicinal, por não apresentarem caráter tóxico. Não necessitam de elevadas temperaturas, ou de adição de catalisadores na sua produção. A síntese é feita apenas com copolímero tribloco Pluronic F127, e o ácido cloroáurico tri-hidratado $\left(\mathrm{HAuCl}_{4} \cdot 3 \mathrm{H}_{2} \mathrm{O}\right)$; gerando um produto ambientalmente amigável, estável e limpo ${ }^{1} \mathrm{O}$ presente trabalho apresenta-se como alternativa simples e eficaz para interpretação dos dados de espalhamento dinâmico de luz de nanopartículas de ouro sintetizadas com Pluronic F127 em temperatura ambiente evitando-se a necessidade de alteração do nanocompósito com diluições.

\section{Metodologia}

Os reagentes utilizados neste trabalho, fornecidos pela Sigma-Aldrich (Brasil) e usados sem purificação adicional foram: o copolímero tribloco Pluronic F127 (12.600 g.mol $\left.{ }^{-1}\right)$ e o ácido cloroáurico tri-hidratado $\left(\mathrm{HAuCl}_{4} \cdot 3 \mathrm{H}_{2} \mathrm{O} ; 393,83 \mathrm{~g} \cdot \mathrm{mol}^{-1}\right)$. As soluções de $\mathrm{F} 127\left(0,1\right.$ a $\left.10 \mathrm{mmol} . \mathrm{L}^{-1}\right)$, e a de $\mathrm{HAuCl}_{4}\left(2 \mathrm{mmol} . \mathrm{L}^{-1}\right)$ foram preparadas com água ultra-pura de resistividade de $18 \mathrm{M} \Omega . \mathrm{cm}$ fornecida por um purificador Millipore (Mili-Q). Para o estudo da viscosidade absoluta das soluções de F127 o tempo de escoamento de cada solução e do líquido padrão (água), foi medido em um viscosímetro de Ostwald a $25^{\circ} \mathrm{C}$, utilizando um cronômetro. Após medição, obteve-se o tempo médio para cada concentração, e determinou-se a densidade de cada solução polimérica e da água utilizando a massa de um volume fixo $(1 \mathrm{~mL})$. Com os dados de tempo de escoamento ( $t_{F 127}$ e tágua $)$ e densidade ( $\rho_{F 127}$ e $\rho_{\text {água }}$ ) das soluções de F127 e da água (líquido padrão), a viscosidade absoluta $\left(\mu_{\mathrm{abs}}\right)$, em unidades de $\mathrm{cP}$, pode ser determinada pela equação $(1)^{2}: \mu_{\mathrm{abs}}=\mu_{\text {água }}\left(\mathrm{t}_{\mathrm{F} 127} \rho_{\mathrm{F} 127}\right) /$ tágua $\rho_{\text {água }}(1)$.

Para a síntese dos nanocomplexos AuNP-F127 foi adotado o protocolo de Sakai et $\mathrm{al}^{3}$ onde mistura-se soluções de F127 (variando concentração) e $\mathrm{HAuCl}_{4}$ (fixa 2 mmol. $\left.\mathrm{L}^{-1}\right)$ numa proporção em volume de 10:1 (F127:HAuCl 4$)$ mantidas em repouso sob luz e temperatura ambiente $\left(23^{\circ} \mathrm{C}\right)$ até a formação das nanopartículas de ouro por até 4 horas. Os valores de diâmetro hidrodinâmico $\left(\mathrm{D}_{\mathrm{H}}\right)$ dos nanocomplexos AuNPF127 foram medidos utilizando um equipamento Zetasizer NanoZS (Malvern Instruments) com análise da radiação espalhada sob ângulo de $173^{\circ}$ à $25^{\circ} \mathrm{C}$.

\section{Resultados e Discussão}

Nanopartículas de ouro foram sintetizadas em várias concentrações de F127 pelo método Sakai et $\mathrm{al}^{3}$. Ao caracterizar os nanocomplexos AuNP-F127 por espalhamento dinâmico de luz um abrupto aumento foi observado nos valores do $\mathrm{D}_{\mathrm{H}}$, que para 
nanocomplexos sintetizados com 10 mmol.L $^{-1}$ de F127 ultrapassou $300 \mathrm{~nm}$, como mostra a Fig 1 (b). Então, a viscosidade absoluta ( $\left.\mu_{\text {abs }}\right)$ das soluções de F127 foi determinada pela equação 1 e um crescimento exponencial foi observado com o aumento da concentração de F127 (Fig. 1 (a)). Esse comportamento é causado pela resistência interna das soluções de F127 ao escoar pelo capilar proporcionados pelo aumento da quantidade de polímero em solução. Ao utilizarmos esse valor de viscosidade no software da Malvern as medidas de $\mathrm{D}_{\mathrm{H}}$ foram corrigidas e o resultado encontrado está apresentado na Fig. 1 (c).
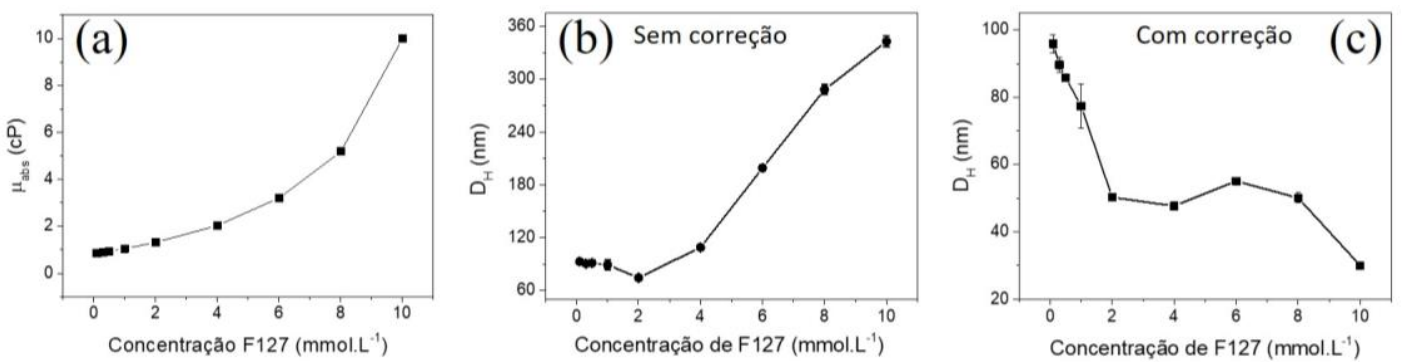

Fig. 1. Viscosidade absoluta das soluções de F127 em função da concentração de polímero a $25^{\circ} \mathrm{C}$. Diâmetro hidrodinâmico dos nanocomplexos em função da concentração de F127: (a) sem diluição utilizando a viscosidade da água e em (b) utilizando a viscosidade do meio obtida em (a).

Desta forma, os valores de $\mathrm{D}_{\mathrm{H}}$ dos nanocompósitos são mais reais confirmando o resultado obtido na Fig 1. Essa alteração nos valores de $\mathrm{D}_{\mathrm{H}}$ ocorreu devido a relação inversa entre a viscosidade do meio e o $\mathrm{D}_{\mathrm{H}}$ dada pela equação de Einsten-Stokes ${ }^{4}$.

\section{Conclusões}

Os dados de viscosidade das soluções de F127 foram utilizados para corrigir a discrepância encontrada nos dados de $\mathrm{D}_{\mathrm{H}}$ dos nanocomplexos AuNp-F127 ( 300 nm para F127> $8 \mathrm{~nm}$ ). Com esta correção os novos valores encontrados para $\mathrm{D}_{\mathrm{H}}$ permanecem próximos de $50 \mathrm{~nm}$ quando o regime micelar é atingido mantendo praticamente constante. Isto sugere que micelas de F127 $\left(\mathrm{D}_{\mathrm{H}} \sim 40 \mathrm{~nm}\right)$ aumentam de tamanho para acomodar as AuNP's. Dados apresentados na literatura $\left(2 \mathrm{mmol} . \mathrm{L}^{-1} \mathrm{de}\right.$ F127) após diluição deste sistema em água apresentam $D_{H} \sim 42 \mathrm{~nm}^{5}$. Desta forma, este trabalho permite uma interpretação mais autêntica do nanocomplexo polimérico AuNP-F127 abrangendo em uma ampla faixa de concentrações de polímero.

\section{Agradecimentos}

Agradecemos ao CNPq, a CAPES, a FAPERO, e aos técnicos e professores do Lab. de Ens. de Físico-Química do IQ-UnB.

\section{Referências}

${ }^{1}$ T. Sakai, P. Alexandridis, Single-step synthesis and stabilization of metal nanoparticles in aqueous pluronic block copolymer solutions at ambient temperature, Langmuir. 20 (2004) 8426-8430

${ }^{2}$ CRC Handbook of Chemistry and Physics; 58th Edition, CRC Press, Inc., Cleveland, Ohio; Editor: Robert C. Weast; 1977-1978.

${ }^{3}$ T. Sakai, P. Alexandridis, Mechanism of gold metal ion reduction, nanoparticle growth and size control in aqueous amphiphilic block copolymer solutions at ambiente conditions, J. Phys. Chem. B. 109 (2005) 7766-7777.

${ }^{4}$ Berne, J. B. \& Pecora, R. Dynamics Light Scattering. (DOVER SCIENCE, 2000).

${ }_{5}^{5}$ Simon, T., Boca, S. C. \& Astilean, S. Pluronic-Nanogold hybrids: Synthesis and tagging with photosensitizing molecules. Colloids Surfaces B Biointerfaces. 97 (2012), 77-83. 